\title{
Relative Therapeutic Efficacy of the Treadmill and Step Bench in Gait Rehabilitation of Hemiparetic Stroke Patients
}

\author{
Badaru U.M.,' Alonge V., ${ }^{2}$ Adeniyi A.F., ${ }^{3}$ Ogwumike 0.0. ${ }^{3}$ \\ ${ }^{1}$ Department of Physiotherapy, Faculty of Medicine, Bayero University, Kano, Nigeria \\ ${ }^{2}$ Department of Physiotherapy, Aminu Kano Teaching Hospital, Kano \\ ${ }^{3}$ Department of Physiotherapy, College of Medicine, University of Ibadan, Ibadan, Nigeria \\ Correspondence \\ Umaru M. Badaru•E-mail badaruum@yahoo.com
}

\begin{abstract}
Summary
The aim of this research is to compare the efficacy of treadmill and step bench exercises in hemiparetic gait rehabilitation. Previous studies have supported the use of treadmill and step bench exercises in gait rehabilitation.

Nineteen patients were recruited for an 8-week, 2 -group quasi-experimental study which was conducted at the Aminu Kano Teaching Hospital. The patients were randomly distributed into 2 groups: A (step bench exercise) and B (treadmill). Groups A and B had 9 and 10 participants respectively. The mean age of the participants in group A was $47.78 \pm 8.17$ years, while that of participants in group B was $46.9 \pm 7.11$ years. Data collected on cadence, stride length, stride width, step length, stride velocity and foot angle before treatment at the $4^{\text {th }}$ and $8^{\text {th }}$ weeks of treatment were analysed using inferential statistics.

There was a significant difference $(\mathrm{p}<0.05)$ in the pre-treatment and post-treatment scores in both groups. Comparison of the post-treatment mean scores of the 2 groups was made and the $t$-values for cadence, stride length, step length, stride width, stride velocity and foot angle were 1.18, -0.91,-0.86, 0.78,1.19 and 0.01, respectively, after analysis was carried out using the unpaired t test. The results show ed no significant difference in the mean scores of the measured gait parameters for the groups $(p>0.05)$.

It was concluded that hemiparetic stroke patients will benefit from both rehabilitation protocols, and that neither rehabilitation protocol is superior to the other.
\end{abstract}

KEY WORDS: Hemiparesis, gait, rehabilitation, treadmill, step-bench

\section{INTRODUCTION}

Stroke gait relative to normal value is characterized by low velocity, low cadence, short stride length, increased double support phases and asymmetric single limb loading (ElBahrawy and El- Tamawy, 2004). In rehabilitation, a lot of time is spent on gait re-education and recovery of mobility (Gladman et al, 1993). The ability to walk is seen as a highly desired goal among stroke survivors, and it is used as a yardstick for measuring recovery by both patients and their relatives (Hamzat and Olaleye, 2002; Patterson et al,
2008). Macko et al (2005) reported that treadmill exercise improves both functional mobility and cardiovascular fitness in patients with chronic stroke. Treadmill exercise improves a 30 -foot timed walk by $17 \%$, 6-minute walk by $13 \%$, unassisted walking velocity by $22 \%$, stride length by $13 \%$ and cadence by 7\% (Patterson et al, 2008). Treadmill training with partial body weight support (PBWS) may produce better walking and postural abilities than gait training on full weight (Pomeroy and Manning 2003; Barbeau and Visintin, 2003). Pohl et al (2002) showed that 
structured speed-dependent treadmill training after a stroke incidence resulted in better walking ability than limited progressive treadmill training and conventional gait training.

Step exercise was shown to increase gait performance in the elderly (Nishimoto et al, 1999). It can also be used to measure aerobic fitness in apparently healthy subjects (Akinyele, 2007). Step bench exercise trains stroke patients to initiate gait with the paretic lower limb. Weight bearing through or 'loading' of the paretic lower extremity and transfer of weight from one lower extremity to the other are important impairment-level goals of stroke rehabilitation (Mercer et al, 2009). Besides, Hesse et al (1997) found that when hemiparetic patients initiate gait with the affected leg, the movement pattern of the centre of pressure mass was comparable to that of the normal gait. Loading of the paretic lower extremity relates to the performance of many functional tasks including walking (Mercer et al, 2009). The objective of this research is to compare the efficacy of treadmill and step bench exercises in hemiparetic gait rehabilitation.

\section{METHODOLOGY}

The materials used for the study include a roll of white paper ( 3 metres long, 1/2 metre wide), 2 bottles of ink of different colours, masking tape, a stopwatch, a sphygmomanometer and a stethoscope, a treadmill (Healthcare, England) and a step bench (8 inches high, 14 inches long, 13 inches wide). Nineteen patients were recruited for an 8-week, 2-group quasi-experimental study which was conducted at the Aminu Kano Teaching Hospital, Nigeria. The patients were randomly distributed into 2 groups: A (step bench) and B (treadmill), with 9 and 10 participants, respectively. The patients were educated about the exercise and their blood pressures were measured before the commencement of every exercise session. The step bench exercise was done at 4 counts:

i. Up with one foot (the affected leg);

ii. Up with the second foot, body erect, legs straight on the bench;

iii. Down with the same foot placed on the bench first;

iv. Down with the other foot.

The treadmill group walked at a comfortable treadmill speed. All treatment sessions lasted between 4 and 10 minutes, with rest periods in between each session. There were three treatment sessions per week for eight weeks. The data collection procedure was adopted from Boenig
(1977). Two moleskin tapes - one triangular and one square - saturated with ink were placed at the midline of the sole of each patient's shoes (front and heel). The moleskin tapes on each shoe were saturated with ink of different colours. The patient was then instructed to walk on the white paper that was taped to the floor. A stopwatch was used to record the time needed for the patient to walk across the entire length of the paper. The data collected on cadence, stride length, stride width, step length, stride velocity and foot angle before treatment, and at the $4^{\text {th }}$ week and $8^{\text {th }}$ weeks of treatment were analysed using inferential statistics.

\section{Cadence}

Cadence is the measurement of the number of steps taken per unit time. The value was calculated by dividing the total number of steps taken on the paper by the total time needed to walk across the length of the paper (measured in steps per minute).

\section{Stride Length}

The midpoint of the heel square was used as reference point for measurement. The stride length is the linear distance from the heel strike of one foot to the heel strike of the next successive step of the same foot (Distance AB measured in centimetres - figure 1).

\section{Step Length}

This is the measurement of the distance from the heel strike of one foot to the heel strike on the next successive step of the opposite foot, using the midpoint of the heel square as the reference point (Distance DC measured in centimetres - figure 1).

\section{Step Width (Stride width)}

Step width is the transverse linear distance between points on 2 successive feet. Heel-to-heel step width was calculated as the difference between distance $\mathrm{G}$ and $\mathrm{H}$ (figure 2), measured from the midpoint of 2 successive heel squares of the opposite feet to the edge of the paper. Toe-to-toe width was calculated in the same manner using the apices of the triangles as the reference point for measurement (E minus F). The average of the two measurements gives the stride width in centimetres.

\section{Stride Velocity}

Stride velocity is a measure of the distance covered per unit time. 
Relative Therapeutic Efficacy of the Treadmill and Step Bench in Gait Rehabilitation of Hemiparetic Stroke Patients

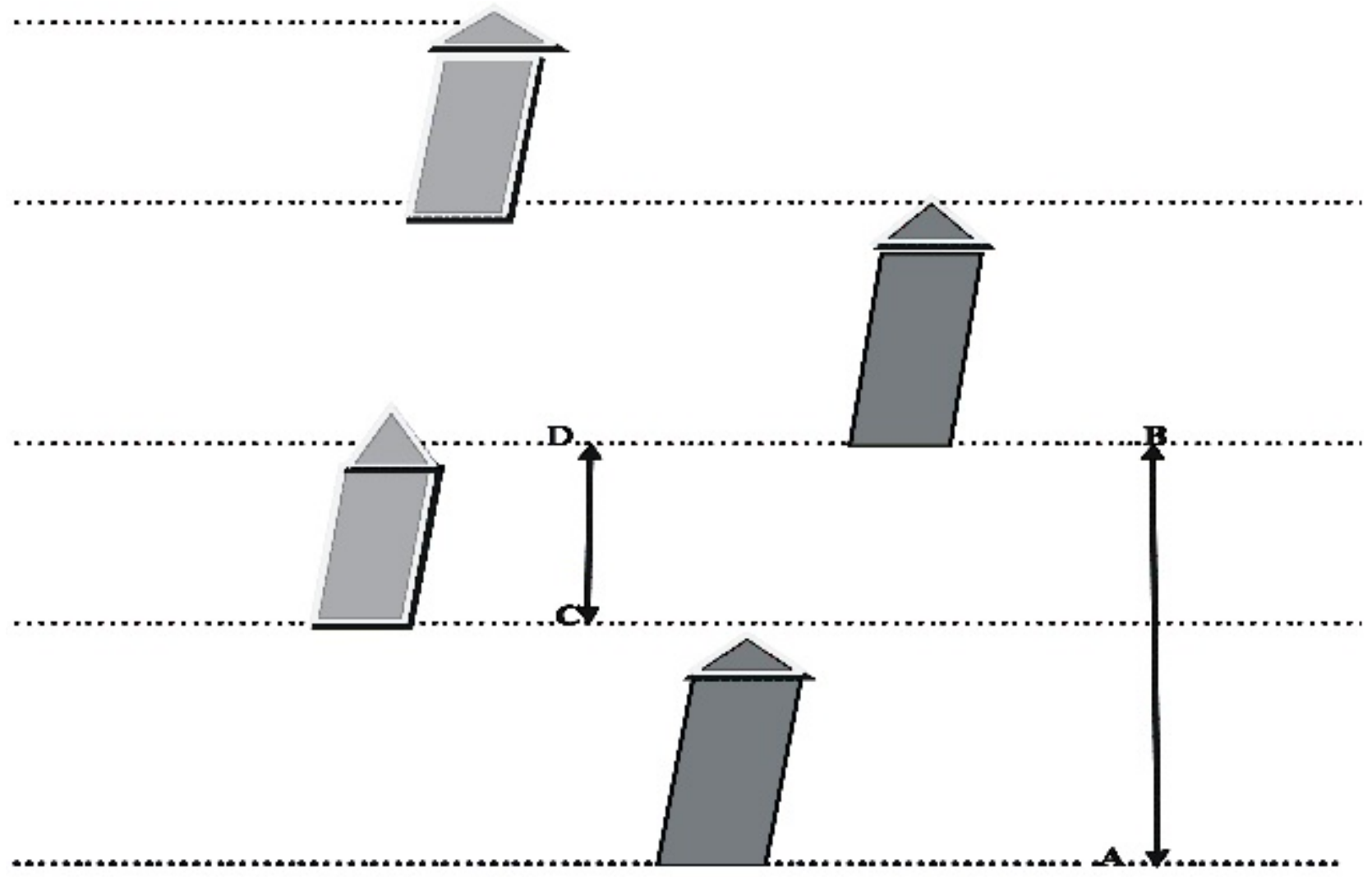

Figure 1. Measurement of stride length (distance $A B$ ) and step length (distance $C D$ ) from imprint on the paper (adopted from Boenig, 1977)

$$
=\text { toe }
$$

$$
=\text { heel }
$$
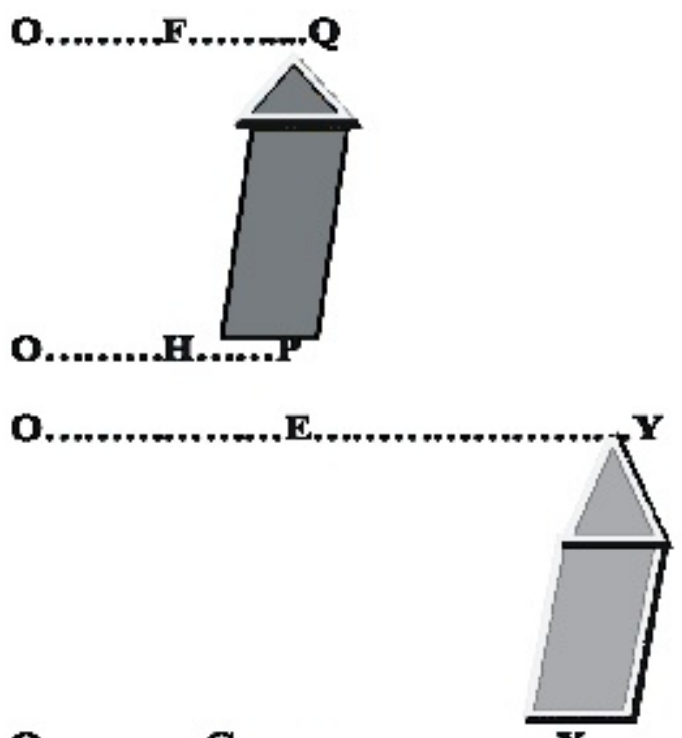

O.................................

Figure 2. Measurement of stride width from the imprint on the paper strip (Boenig, 1977) 


\section{Foot Angle}

Foot angle refers to the amount of toe-out, toe-in of each foot. For each step, a long axis was drawn through the apex of the toe triangle and the midpoint of the heel square. A line intersecting with the long axis was drawn perpendicular to the line of progression. A protractor placed on the perpendicular line, intersecting with the long foot axis was used to determine the angle of toe-out or toe-in (figure 3).

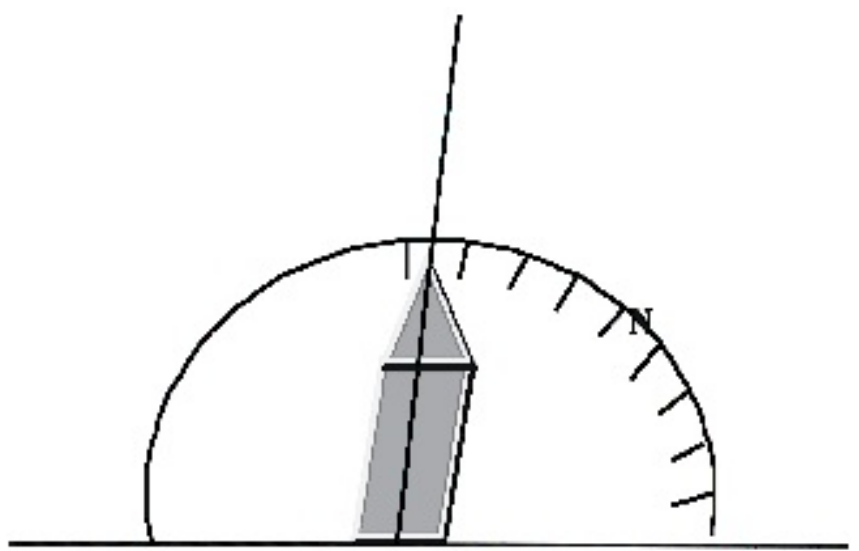

Figure 3. Measurement of foot angle from the imprint on the paper stip (Boenig, 1977)

\section{RESULTS}

The participants comprised 14 males $(73.7 \%)$ and 5 females $(26.32 \%)$. Group A $(n=9)$ had 7 males and 2 females and their ages ranged from 40 to 62 years, while 7 males and 3 females within the age range of 37 to 60 years formed group $B(n=10)$. The mean age of participants in group $A$ was $47.78 \pm 8.17$ years, while that of group B was $46.9 \pm$ 7.11 years Most of the participants were businessmen $(52.6 \%)$ and housewives $(26.32 \%)$. Civil servants and farmers constituted $10.53 \%$ each.

The difference between the pre-treatment and posttreatment values of gait parameters in groups A and B is presented in table 1 . The results were analysed using the paired t-test and found to be significant for both groups ( $\mathrm{P}$ $<0.05)$. The post-treatment mean scores for the two groups were compared. The results showed that the cadence mean score for group A $(90.04 \pm 8.67$ steps/minute $)$ and $\mathrm{B}(84.08 \pm 13.02$ steps/minute $)$, the stride length score for group A $(62.05 \pm 20.18 \mathrm{~cm})$ and B $(69.64 \pm 15.50 \mathrm{~cm})$, the step length score for group A $(31.66 \pm 8.86 \mathrm{~cm})$ and B $(34.96 \pm 7.8 \mathrm{~cm})$, the stride width score for group A $(13.01 \pm 3.99 \mathrm{~cm})$ and $B(11.70 \pm 3.32 \mathrm{~cm})$, the stride velocity score for group A $(0.64 \pm 0.06 \mathrm{~m} / \mathrm{s})$ and B $(0.6 \pm$
$0.09 \mathrm{~m} / \mathrm{s})$, and the foot angle score for group A $(9.72 \pm$ $\left.3.62^{\circ}\right)$ and $\mathrm{B}\left(9.63 \pm 3.96^{\circ}\right)$ were all not significant when analysed using the unpaired $\mathrm{t}$ test as presented in table 2 .

Table 1. Comparison of the difference between pre-treatment and post-treatment mean values within groups

\begin{tabular}{|c|c|c|c|}
\hline Group & $\begin{array}{r}\text { Pre-treatment } \\
\text { Mean } \pm \text { S.D }\end{array}$ & $\begin{array}{l}\text { Post-treatment } \\
\text { Mean } \pm \text { S.D }\end{array}$ & $\begin{array}{l}\text { Calculated } \\
\mathrm{t}\end{array}$ \\
\hline \multicolumn{4}{|l|}{ A $\quad(N=9)$} \\
\hline Cadence & $\begin{array}{l}78.17 \pm 14.57 \\
\text { steps/min. }\end{array}$ & $\begin{array}{l}90.04+8.67 \\
\text { steps/min. }\end{array}$ & $-3.33 *$ \\
\hline Stride width & $15.26+5.04 \mathrm{~cm}$ & $13.01+3.99 \mathrm{~cm}$ & $3.93 *$ \\
\hline Step length & $27.68+8.71 \mathrm{~cm}$ & $31.66+8.86 \mathrm{~cm}$ & $-3.13 *$ \\
\hline Stride velocity & $0.56 \pm 0.1 \mathrm{~m} / \mathrm{s}$ & $0.64 \pm 0.06 \mathrm{~m} / \mathrm{s}$ & $-3.337 *$ \\
\hline Foot angle & $0.84 \pm 4.29^{\circ}$ & $9.72 \pm 3.62^{\circ}$ & $3.37 *$ \\
\hline $\begin{array}{l}\text { Stride length } \\
\text { B } \quad(N=10)\end{array}$ & $56.46+18.3 \mathrm{~cm}$ & $62.05+20.18 \mathrm{~cm}$ & $-2.92 *$ \\
\hline Cadence & $\begin{array}{l}75.48+13.99 \\
\text { steps/min. }\end{array}$ & $\begin{array}{l}84.08+13.02 \\
\text { steps/min. }\end{array}$ & $-5.1 *$ \\
\hline Stride width & $13.56 \pm 3.22 \mathrm{~cm}$ & $11.70 \pm 3.21 \mathrm{~cm}$ & $3.65 *$ \\
\hline Step length & $31.84 \pm 9.1 \mathrm{~cm}$ & $34.96 \pm 7.8 \mathrm{~cm}$ & $-3.67 *$ \\
\hline Stride velocity & $0.54+0.1 \mathrm{~m} / \mathrm{s}$ & $0.6 \pm 0.09 \mathrm{~m} / \mathrm{s}$ & $-5.09 *$ \\
\hline Foot angle & $10.85 \pm 4.17^{\circ}$ & $9.63 \pm 3.96^{\circ}$ & $4.88^{*}$ \\
\hline Stride length & $63.21+18.07 \mathrm{~cm}$ & $69.64+15.50 \mathrm{~cm}$ & $-4.89 *$ \\
\hline
\end{tabular}

Table2. Comparison of post-treatment means scores of gait parameters across the groups

\begin{tabular}{|c|c|c|c|}
\hline $\begin{array}{l}\text { Gait } \\
\text { parameters }\end{array}$ & $\begin{array}{c}\text { A } \\
\text { Mean } \pm \text { S.D } \\
(\mathrm{N}=9)\end{array}$ & $\begin{array}{c}\text { B } \\
\text { Mean } \pm \text { S.D } \\
(\mathrm{N}=10)\end{array}$ & $\begin{array}{l}\text { Calculated } \\
\mathrm{t}\end{array}$ \\
\hline Cadence & $\begin{array}{l}90.04 \pm 8.67 \\
\text { steps/min. }\end{array}$ & $\begin{array}{l}84.08+13.02 \\
\text { steps/min. }\end{array}$ & 1.18 \\
\hline Stride length & $62.05+20.18 \mathrm{~cm}$ & $69.64+15.50 \mathrm{~cm}$ & -0.91 \\
\hline Step length & $31.66 \pm 8.86 \mathrm{~cm}$ & $34.96 \pm 7.8 \mathrm{~cm}$ & -0.86 \\
\hline Stride width & $13.01 \pm+3.99 \mathrm{~cm}$ & $11.70 \pm 3.32 \mathrm{~cm}$ & 0.78 \\
\hline Stride velocity & $0.64 \pm 0.06 \mathrm{~m} / \mathrm{s}$ & $0.6 \pm 0.09 \mathrm{~m} / \mathrm{s}$ & 1. 19 \\
\hline Foot angle & $9.72+3.62^{\circ}$ & $9.63 \pm 3.96^{\circ}$ & 0.01 \\
\hline
\end{tabular}

\section{DISCUSSION}

The results of the comparison of the pre-treatment and post-treatment scores for each group showed significant improvement of the gait parameters using step bench and treadmill exercises. This implies that step bench and treadmill exercises are rehabilitation protocols that can significantly improve the gait pattern of hemiparetic stroke patients. These results were in agreement with the findings of previous studies. For example, improvements in 
cadence, stride length and stride velocity agreed with the findings of Patterson et al (2008) and Pohl et al (2002), who reported that structured speed-dependent treadmill training resulted in better walking ability than the controls. These results were also in line with the report of Macko et al (2005) that treadmill exercise improves functional mobility in patients with chronic stroke. The improvement in gait ability after step bench exercise and hemiparetic limb loading was in consonance with the findings of Nishimoto et al (1999). It also agreed with the findings of Mercer et al (2000) and Hesse et al (1997), who found that loading of the paretic lower extremity relates to the improved performance of many functional tasks including walking, which also improved weight, equal weight transference and symmetry of gait. The result of the comparison of the posttreatment mean scores for both groups showed no significant difference. This implies that there is no statistically significant difference in the efficacy of both modalities in the rehabilitation of the gait of hemiparetic stroke patients.

\section{CONCLUSION}

The findings of this study have shown that step bench exercises and treadmill retraining are beneficial in the rehabilitation of the gait of hemiparetic stroke patients. It was concluded that step bench exercises and treadmill retraining are both beneficial, and that neither is superior to the other. It was recommended that further research be carried out to determine how effective gait rehabilitation will be if these modalities are combined.

\section{References}

Akinyele, O.D. 2007. Development of A New Step Test Protocol for Cardiorespiratory Fitness Assessment. An Unpublished M.Sc. Dissertation Submitted to Faculty of Clinical Sciences, College of Medicine, University of Ibadan.

Barbeau, H. and M. Visintin. 2003. Optimal outcomes with body weight support combined with treadmill training in stroke patients. Archive of Physical Medicine and Rehabilitation 84: 1458-1465.
Boenig, D.D. 1977. Evaluation of clinical method of gait analysis. Physical Therapy 57(7): 795-798.

El-Bahrawy, E.N. and M.S. El-Tamawy. 2004. Spasticity control and its relation to foot print in stroke patients. Egyptian Journal of Neurology, Psychiatry and Neurosurgery 14(1): 295-302.

Gladman, J., N. Lincoln and D. Barer. 1993. A randomized controlled trial of domiciliary and hospital-based rehabilitation for stroke patients after discharge from hospital. Journal of Neurology, Neurosurgery and Psychiatry 55: 960-966

Hamzat, T.K. and O.A. Olaleye. 2002. Stroke rehabilitation: When should ambulation commence. Journal of Nigeria Medical Rehabilitation Therapists 7(20) 23-25.

Hesse, S., F. Reiter, M. Jahnke, M. Dawson, T. Sarkodie-Gyan and K.H. Mauritz. 1997. Asymmetry of gait initiation in hemiparetic stroke subjects. Archive of Physical Medicine and Rehabilitation 78(7): 719-24.

Macko, R.F., F.M. Ivey, L.W. Forrester, D. Hanley, J.D. Sorkin, L.I. Katzel, K.H. Silver and A.P. Goldberg. 2005. Treadmill rehabilitation improves ambulatory function and cardiovascular fitness in patients with chronic stroke. Stroke 36: 2206-2221.

Mercer, V.S., J.K. Freburger, S. Chang and J.L. Purser. 2009. Measurement of paretic-lower-extremity loading and weight transfer after stroke. Physical Therapy 89(7): 653-664.

Nishimoto, K., S. Nakamura, T. Imai, S. Tanaka and S. Fujimoto. 1999. Increasing gait performance in elderly women using a step exercise training program. Journal of Physical Therapy Science 11: 71-78.

Patterson, S.L., M.M. Rodgers, R.F. Macko and L.W. Forrester. 2008. Effect of treadmill exercise training on spatial and temporal gait parameters in subjects with chronic stroke: A preliminary report. Journal of Rehabilitation Research and Development 48(2) 221-228.

Pohl, M., J. Mehrholz., C. Ritschel.and S. Rückriem. 2002. Speed-dependent treadmill training in ambulatory hemiparetic stroke patients: A randomized controlled trial. Stroke 33: 553558.

Pomeroy, V.M. and C.D. Manning. 2003. Effectiveness of treadmill retraining on retraining of gait of hemiparetic stroke patients: A systematic review of current evidences. Physiotherapy 89(6) 337-349. 